\title{
Soft Computing Techniques for Edge Detection Problem: A state-of-the-art Review
}

\author{
Naveen Singh Dagar \\ Research Scholar, \\ Electronics \& Communication Engg. Dept., \\ Deenbandhu Chhotu Ram University of Science\& \\ Technology, Murthal, INDIA-131039.
}

\begin{abstract}
Edge is an abrupt change that occurs in an image. Edge detection is one of the most prevalent problems in image processing. Edge Detection is the approach used most frequently for segmenting images based on abrupt changes in intensity. It is a concept that covers a number of fields in today's environment. In this paper, a state-ofthe-art review of the conventional Edge Detection Techniques is presented. The paper also presents a stateof-the-art review of Soft Computing Techniques such as Fuzzy Logic, Genetic Algorithm, Neural Networks, Evolutionary Computation, Swarm Intelligence etc. for Edge Detection Problem. Further, an analysis of the review is also presented.
\end{abstract}

\section{Keywords}

Edge, Hysteresis Threshold Methods, Image Segmentation, Otsu, Fuzzy Logic, Soft Computing, Genetic Algorithm, Swarm Intelligence, Evolutionary Computation, Neural Network.

\section{INTRODUCTION}

Edge Detection is one of the upcoming concepts used in various fields for the detection of edge especially in fields using images for studying medical, biological, astrological, industrial and other fields. The image is basically segmented using the four methods, Smoothing/Filtering, Enhancement, Detection, and Localization [1]. Any image before being given to any of the Edge Detection Techniques are to be Smoothed/Filtered to reduce the occurrences of salt and pepper noise in the image and other noises such as Gaussian noise and impulse noise, enhanced to facilitate better Edge Detection by determining the changes in intensity, detected in order to separate the edges, and localized [1].

In a technical view the Edge Detection Technique may be a search based procedure detecting the edges by checking for the local maxima along the direction matching the edge profile or a zero crossing technique that selects the second order derivative expression computed which may be the Laplacian or the non linear differential expression. Similarly, from a conceptual point of view the edge detection technique can be classified as a contextual or a non-contextual approach [2]. Once the image is smoothed and enhanced the edges are segmented using various algorithms. The algorithms which may be classical augmented or may be a derived algorithm [26].

Edge Detection is performed in various fields using the appropriate methods [2]. The result of applying an edge detector leads to a set of connected components that indicate the boundaries. Thus, applying the Edge Detection algorithm to an image may significantly reduce
Pawan Kumar Dahiya

Assistant Professor, Electronics \& Communication Engg. Dept., Deenbandhu Chhotu Ram University of Science\& Technology, Murthal, INDIA-131039.

the amount of data to be processed leading to ignoring the irrelevant data and capturing the important structural properties of the image [3]. If the Edge Detection step is successful, the subsequent task of interpreting the information contents in the original image may be simplified. Still the possibility of obtaining such ideal edges from real life images is complex. Edge Detection is one of the fundamental steps in image processing, image analysis, image pattern recognition, and computer vision [26].

The Classical Edge Detection Techniques involves different operators such as Prewitt operator, Sobel operator, Robert Cross edge operator, Robinson, and FreiChen. The Gaussian based method includes some optimal Edge Detection methods such as Laplacian of Gaussian (LoG) edge detection, Canny Edge Detection and the ISEF algorithm. The Soft Computing Approaches such as Fuzzy based Edge Detection technique, Morphology based multi structure elements Edge Detection, Genetic Algorithm, Neural Network based approach.

The contribution of the paper is as under:

- The paper presents a state-of-the-art review of Edge Detection Techniques.

- The paper presents a state-of-the-art review of Soft Computing Techniques for Edge Detection Problem.

- The paper, further, presents an analysis of the review of the above.

The rest of the paper is organized as under:

Section II details the Edge Detection problem and a review of Edge Detection Techniques. Section III details the Soft Computing Techniques. Section IV reviews the Soft Computing Techniques for Edge Detection Problem. Section V presents an analysis of the review. Section VI concludes the paper.

\section{EDGE DETECTION PROBLEM AND EDGE DETECTION TECHNIQUES}

\subsection{Classical method}

Edge Detection based on computing the gradient for the pixels and detecting the local maxima to localize the step edges.

$$
\left|\widehat{\nabla_{\mathrm{g}}}(\mathrm{c}, \mathrm{r})\right|=\left|\sum_{\alpha} \mathrm{g}_{\alpha}(\mathrm{c}, \mathrm{r})\right|
$$

It is a basic method for the detection of the edges and their orientation [5]. The technique being simple is based radically on the discrete differential operators. The Sobel edge detector is a simple and an effective operator but is 
disturbed by noise easily and is not useful in detecting the outermost edges clearly. The Prewitt operator does not provide the smoothing provided by the Sobel operator. The major drawback seen in these methods is that it is sensitive to noise and is not accurate.

\subsubsection{Roberts Cross Edge Detector}

The Robert Cross Edge Detector is based on the 2D spatial gradient measurement of the image. The Edge Detection is performed by the high spatial frequencies [5]. The magnitude of the spatial gradient of the input image for each different pixel is provided as the output for each pixel, a gray scale image. The convolution kernel used is as shown in the Figure1. Robert proposed the equation:

$$
\begin{gathered}
y_{i, j}=\sqrt{x_{i, j}} \\
z_{i, j}=\sqrt{\left(y_{i, j}-y_{i+1, j+1}\right)^{2}+\left(y_{i+1, j}-y_{i, j+1}\right)^{2}}
\end{gathered}
$$

where $\mathbf{x}$ - Initial intensity of the image

$\mathbf{z}$ - Derivative computed. $\mathbf{i}, \mathbf{j}$ - location in the image.
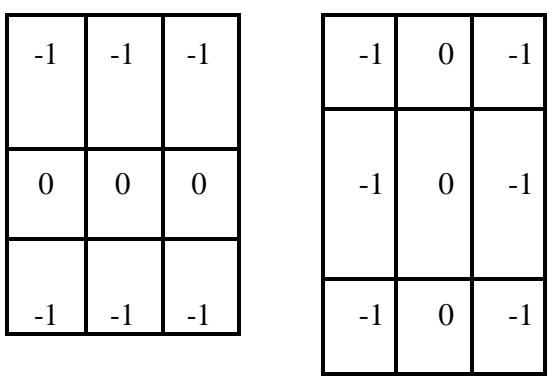

Fig 1: Convolution kernels for Robert Edge detection

\subsubsection{Sobel Edge Detection}

The method is similar to the Roberts operator. It finds the approximate absolute gradient magnitude at each point. Here the operator consists of $3 \times 3$ convolution kernels. One kernel is the other rotated by 90 degree. Finally, the gradient magnitude is thresholded.

$$
|G|=\sqrt{G_{x}^{2}+G_{y}^{2}}, q=\arctan \left(\frac{G_{x}}{G_{y}}\right)
$$

\subsubsection{Prewitt Edge Detection}

The Prewitt edge detection technique was given to overcome the problem faced in Sobel Edge Detection due to the absence of the smoothing modules. The operator adds a vector value in order to provide smoothing [18]. This operator or algorithm provides a way to estimate both the magnitude and the orientation of an edge.

$$
|G|=\sqrt{G_{x}^{2}+G_{y}^{2}} \theta=\operatorname{atan} 2\left(G_{x}, G_{y}\right)
$$

It involves both the horizontal and the vertical edge positions. It is limited to 8 possibilities of orientation. The convolution mask with the largest module is now selected [18].

\subsection{The optimal Edge Detection Technique (Gaussian Method)}

The method given by Marr-Hilderth experiment the concept, that an edge corresponds to a change in the image orientation, thus informing that it is much intense to find the zero crossing position than the maxima. Due to the use of the Laplacian filter, the corners and curves are not detected accurately. To overcome these disadvantages the improved methods, the Canny and the ISEF methods of the optimal Edge Detection technique is used [20]. The Canny operator analysed the product with the Gaussian first order derivative to identify the boundary. The possibility of pixels at the boundary being noise was identified. This leads to the ISEF algorithm (Shen and Castan's filter) providing better signal to noise ratio.

\subsubsection{Laplacian of Gaussian (LoG) edge detection}

The major improvement is seen over in the noise reduction. A 2D Gaussian function is used to convolve the image

$$
\begin{aligned}
& L(x, y)=\nabla^{2} f(x, y)=\frac{\partial^{2} f(x, y)}{\partial x^{2}}+\frac{\partial^{2} f(x, y)}{\partial y^{2}} \\
& \operatorname{LoG}(x, y)=-\frac{1}{\pi \sigma^{4}}\left[1-\frac{x^{2}+y^{2}}{2 \sigma^{2}}\right] e^{-\frac{x^{2}+y^{2}}{2 \sigma^{2}}}
\end{aligned}
$$

The Laplacian is then computed and the pixels with zero crossing are considered as edges [8].

Image pyramid is a method of applying repeated smoothing. The pyramid is based on two filters the low pass filter and the high pass filter.

\subsubsection{Canny Edge Detection}

Considering the white Gaussian Noise a new operator providing a convolution filters to smooth the noise as well as detect the edges more accurately was introduced. Canny Edge detector facilitates three characteristics required for the detection of edges. Error Rate indicating that the detection of Edges must be increased. SNR, the signal to noise ratio must be kept as high as possible. Localization provides the distance between the actual Edge and the detected edge must be as low as possible thus increasing the localization rate. Response formed by the product of the SNR and the localization provides a multiple response [17].

\subsubsection{Infinite Symmetric Exponential Filter}

A similar technique as the Canny Edge Detection, but varying in concept of using the optimal filter, gave rise to the ISEF edge detection technique which was given by Shen and Castan. In Canny the use of the filter with Gaussian derivative is seen, whereas the filter is directly being used thus providing a good localization and detection in ISEF. Even in an image with low signal to noise ratio localization and detection is to the best. Nonmaxima suppression is used for the purpose of thinning.

\subsection{Multi-resolution method}

Schunk, Witkin, BerGholm, Lacroix, William and Shah, Goshtasby, Jeong and Kim, and Deng an dCahill each of them produces different algorithms by combing the classical and the Gaussian forms of detectors mainly to overcome the low signal noise ratio and provide better localization. Finally, Bennamoun gave a hybrid algorithm separating the processes of noise removal and Localization among various detectors.

\subsection{Non Linear Methods}

These methods evolved as a result of the heat equation. Perona and Malik provides this using the anistropic Diffusion Technique. This helps in recognition of the Edges through the Space variant blurring, thus, giving smoothed areas and sharp edges or boundaries. The 
process is being spelled as Discretization. This method provides unstable equation results leading to the concept of Wavelet based Techniques.

\subsection{Wavelet Based Method}

The wavelet based method involves the analysis of different scales of the image in order to differentiate the edges of high contrast from the low contrast texture or regions of low contrast. It is defined as a "mathematical microscope" which monitors the image scales. Here instead of the use of local detector, computational modules are used [9]. The spatial transformation between the source image and the edge detected image i.e. the target image is calculated to find the best alignment. The sum of the squared differences is used as the measure for uniformity. The gradient based filter and the wavelet based method is combined and the proposed method is given as the contextual method that can provide more accuracy for the multi-scale resolution images.

\subsection{Statistical Method}

The Statistical method defines the edge detection as a statistical inference. It is a data driven method more than a model based method. Multiple edges cue using multiple scales is combined by joint distribution [11]. The cue calculation provides the relative effectiveness of the detectors for multilevel processing. The four major steps in the process are conditioning, feature extraction, blending, and scaling. Blending the key functions in together acts as the major concepts of computational learning or machine based learning. The algorithm exploits the local signal characteristics and not the thresholding.

\subsection{Contextual method}

The contextual method is a combination of the wavelet method and the gradient based filters. The major algorithm is based on the context of the image [10]. The GAP (Gradient Adjusted Predictor) uses the context which is the combination of the intensity values of the neighbouring pixels. It is found that both the visual evaluation and the objective performance of this method are much superior to Canny and Sobel. Using the cost function the effectiveness of detector is found in presence of noise.

\subsection{Otsu's Method}

An automatic edge detection technique is provided by the Otsu's for image thresholding based on the clustering process. Multi Otsu's method is given by multilevel thresholding [10]. Automatic thresholding involves the knowledge of the object including the intensity and the size of the image, environment the part or the fraction occupied by the object in the image and the applications the number of objects appearing in an image along with their differences. Thresholding that minimizes the weighted class or cluster variances are found [7]. The thresholding is performed directly on the gray level histogram.

\subsection{Line Edge Detector}

Line edge detectors are the local maxima of intensity function in the image. They are mainly used for the detection of roads a nd rivers in the remote sensing images. In best first and the depth first strategy the best first strategy was found to produce best edges. The major problem is that they are not accurate.

\subsection{Colour Edge's Method}

The fundamental difference between the colour images and the gray scale images is that its colour. A colour vector is being used in addition thus considering vector value image than the scalar value images [9]. The two techniques of the monochromatic based using the colour information with colour channels and the colour valued based techniques using the colour vectors. The quantitative and qualitative advantages of the colour edge detection allow the classification of the edges.

\section{SOFT COMPUTING TECHNIQUES}

Soft Computing is a multidisciplinary field that was proposed by Dr. Lotfi Zadeh, with a goal to construct new generation Artificial Intelligence, known as Computational Intelligence [32]. Soft Computing deals with imprecision, uncertainty, partial truth, and approximation to achieve practicability, robustness and low solution cost. Soft Computing in its latest incarnation as the fusion of the fields Fuzzy Logic, Neuro-computing, Evolutionary Computing, Genetic Computing, and Probabilistic Computing. The main goal of Soft Computing is to develop intelligent machines and to solve nonlinear and mathematically un-modeled system problems [6]. Soft computing techniques, which emphasize gains in understanding system behavior in exchange for unnecessary precision, have proved to be important practical tools for many contemporary problems [25]. The Soft Computing Techniques [14] are classified as under:

- $\quad$ Neural networks

- $\quad$ Fuzzy systems

- Evolutionary Computation, including

○ Evolutionary algorithms

- Genetic algorithms

- Differential evolution

○ Metaheuristic and Swarm Intelligence

- Ant colony optimization

- Bees algorithms

- Bat algorithm

- Cuckoo search

- Harmony search

- Firefly algorithm

- Artificial immune systems

- Particle swarm optimization

- Ideas about probability including

○ Bayesian network

- Chaos theory

The applications of Soft Computing have proved two main advantages. First, it made solving nonlinear problems, in which mathematical models are not available, possible. Second, it introduced the human knowledge cognition, recognition, understanding, learning, and others into the fields of computing [25] 
Applying above steps resulted in the possibility of constructing intelligent systems like autonomous selftuning systems, and automated designed systems [6].

\section{SOFT COMPUTING TECHNIQUES FOR EDGE DETECTION PROBLEM}

Soft Computing Techniques [14] exploit the given tolerance of imprecision, partial truth, and uncertainty for a particular problem. Soft Computing Techniques [14] have emerged as potential and robust optimization tools for Edge Detection Problem in recent years [6]. These are summed-up as under:

\subsection{Fuzzy based approaches}

The concept of Fuzzy logic has been extensively applied in characterizing the behaviour of nonlinear systems. The nonlinear behaviour of the system effectively captured and represented by a set of Fuzzy rules [18]. Many engineering and scientific applications including time series are not only nonlinear but also non-stationary. Such applications cannot be represented by simple Fuzzy rules, because fixed number of rules describe time invariant systems only and cannot take in to account the non stationary behaviour. Recently, a new set of Fuzzy rules have been defined to predict the difference of consecutive values of non-stationary time series [19]. The main advantages of Fuzzy Logic approach [20] are that they are easy to understand and build a predictor for any desired accuracy with a simple set of Fuzzy rules. There is no need of mathematical model for estimation and fast estimation of future values due to the less computational demand. The Limitations of Fuzzy Logic approach is that it works on single step prediction and it do not have learning capability.

\subsection{Genetic Algorithm based approaches}

Basically covered by three main processes of selection, cross over and mutation. In selection the fittest pixels are selected. In cross over they are combining to produce a much better pixel. Finally, mutation includes a small change in order to retain the population [6]. It is mainly used with large search spaces with very little knowledge and these clusters of population are estimated to detect the Edge.

\subsubsection{Neural networks based approaches}

The neural networks are low-level computational elements that exhibit good performance when they deal with sensory data. Applied to the situation where there is sufficient observation data available. The Neural network method is used in any problem of control, prediction and classification. Neural Networks are able to gain this popularity because of the commanding capacity that they have in modeling exceptionally complex nonlinear functions. Neural networks have a biggest advantage in terms of easy to use which is based on training-prediction cycles. Training the neural networks plays crucial role in the system usage of neural networks. The training pattern that contains a predefined set of inputs and expected outputs is used to train the neural networks. Next, in prediction cycle, the outputs are supplied to the user based on the input values. To make the neural networks to behave like a physical system or predict or control the training set used in the training cycle shall consist of enough information representing all the valid cases [21, $22,23]$.
Neural Networks are flexible soft computing frameworks for modeling a broad range of nonlinear problems [24]. One significant advantage of the neural network based approach over other classes of nonlinear models is that NNs are universal approximation tools that approximate large class of functions with a high degree of accuracy [25]. This approximation power of Neural Network model comes from several parallel processing elements, called as 'neurons'. No prior assumption of the model form is required in the model building process. Instead, the network model is largely determined by characteristics of the data. Single hidden layer feed forward network is the most widely used model for prediction and forecasting of time variant functions. The model is characterized by a network of three layers of simple processing unit connected by non-cyclic links. The architecture of feedforward neural network is shown [28].

\subsubsection{Ant Colony Optimization based approaches}

The new problem solving approaches that takes inspiration from the social behaviour of insects and other animals is known as Swarm Intelligence. Ant Colony Optimization (ACO) is a nature inspired optimization algorithm [31], motivated by the natural phenomenon that ants deposit pheromone on the ground in order to mark some favourable path that should be followed by other members of the colony. The first ACO algorithm, called the ant system, was proposed by Dorigo et al. [31]. The term "Stigmetry" describes an indirect communication amongst a self-organizing system and it is observed in colonies of ants. When ants are in search of a food resource, they leave a substance called pheromone behind as they move. Other ants perceive the presence of the already deposited pheromone and tend to follow the paths where pheromone concentration is higher [31].

\subsubsection{Evolutionary Computation based approaches}

Evolutionary Computation is a general stochastic search Methodology. Evolutionary computation is becoming popular as it often gives satisfactory results for various optimization problems in different areas. The computation intelligence based CAC use evolutionary approaches like Genetic Algorithm (GA), fuzzy logic and Artificial Neural Networks (ANN). Majority of the computational intelligence based CAC algorithms incorporate fuzzy logic, fuzzy neural and fuzzy MCDM methods. There are very few works reported on the usage of Artificial Neural Networks in CAC [7]. Genetic Algorithm is the most popular evolutionary algorithm. Memetic Algorihm [13] has also been used for Edge Detection Problem.

\subsubsection{Swarm Intelligence based approaches}

Ant colony optimization and Bees algorithm are most popular techniques in swarm intelligence. The Swarm Intelligence based Resource Allocation Algorithm (SIRAA) divides all cognitive radios in a network into sub-networks to reduce the scanning time of each cognitive radio communicates among themselves to get a global view of spectrum availability and their characteristics. The cognitive radios then compute respective bid values and send to the base station. The base station uses binary Particle Swarm Optimization technique for optimal allocation of channels to these radios by maximizing the total bid value [6]. 
Table 1.Review Of Varous Edge Detection Techniques

\begin{tabular}{|c|c|c|c|}
\hline Author & Approach & Advantages & Disadvantages \\
\hline W. Khan et.al. [8] & $\begin{array}{c}\text { Classical } \\
\text { (Robert, Sobel, } \\
\text { Prewitt, Kirsch, ...) }\end{array}$ & $\begin{array}{l}\text { Simplicity, Detection of edges } \\
\text { and their orientations }\end{array}$ & Sensitivity to noise, Inaccurate \\
\hline W. Khan et.al. [8] & $\begin{array}{c}\text { Zero Crossing } \\
\text { (Laplacian, Second } \\
\text { Directional } \\
\text { Derivative) }\end{array}$ & $\begin{array}{l}\text { Detection of edges and their } \\
\text { orientations. Having fixed } \\
\text { characteristics in all directions }\end{array}$ & $\begin{array}{l}\text { Responding to some of the existing } \\
\text { edges, Sensitivity to Noise }\end{array}$ \\
\hline $\begin{array}{l}\text { K.Padma vasavi } \\
\text { et.al. [20] }\end{array}$ & $\begin{array}{c}\text { Laplacian of } \\
\text { Gaussian(LoG) } \\
\text { (Marr-Hildreth) }\end{array}$ & $\begin{array}{l}\text { Find out the correct places of } \\
\text { edges, Tests wider area around } \\
\text { the pixel }\end{array}$ & $\begin{array}{l}\text { Malfunctioning at the curves, corners } \\
\text { and where the intensity of the grey } \\
\text { level function varies, Unable to find } \\
\text { the orientation of edge because of } \\
\text { Laplacian filter }\end{array}$ \\
\hline $\begin{array}{l}\text { K. K. Singh et. Al. } \\
{[21]}\end{array}$ & $\begin{array}{c}\text { Gaussian } \\
\text { (Canny, Shen-Castan) }\end{array}$ & $\begin{array}{l}\text { Using probability for finding } \\
\text { error rate, Localization and } \\
\text { response, Improving signal to } \\
\text { noise ratio, Better detection } \\
\text { specially in noise conditions } \\
\end{array}$ & $\begin{array}{l}\text { Complex Computations, False zero } \\
\text { crossing, Time consuming }\end{array}$ \\
\hline $\begin{array}{l}\text { Anand Gupta et.al } \\
\text { [17], Neveen Singh } \\
\text { Dagar [12] }\end{array}$ & Improvised Canny & $\begin{array}{l}\text { Insensitive to noise, is able to } \\
\text { fabricate superior and accurate } \\
\text { edges in regions of fine graining, } \\
\text { geometrical figures and } \\
\text { alphanumerics images }\end{array}$ & $\begin{array}{l}\text { Complex Computations, Time } \\
\text { Consuming }\end{array}$ \\
\hline N.Sharma et.al.[15] & $\begin{array}{c}\text { Coloured Edge } \\
\text { Detectors }\end{array}$ & $\begin{array}{c}\text { Accurate, } \begin{array}{c}\text { More efficient in object } \\
\text { recognition }\end{array} \\
\end{array}$ & Complicated, Complex Computations \\
\hline $\begin{array}{c}\text { Suchendra M. } \\
\text { Bhandarkar et.al. } \\
{[41]}\end{array}$ & $\begin{array}{l}\text { Gravitational Search } \\
\text { Algorithm }\end{array}$ & $\begin{array}{c}\text { Edges are selected more } \\
\text { smoothly, works acceptably well } \\
\text { on noisy images. }\end{array}$ & $\begin{array}{c}\text { Parameters set correctly to achieve best } \\
\text { possible edge detector for image, } \\
\text { selected features are mistakenly } \\
\text { considered as edge. }\end{array}$ \\
\hline $\begin{array}{l}\text { M.Abdulghafour } \\
\text { et.al. [35] }\end{array}$ & Fuzzy Sets Theory & $\begin{array}{l}\text { Selected features are correctly } \\
\text { considered as edges, less } \\
\text { parameter setting, faster than } \\
\text { other methods, works well on } \\
\text { noisy images. } \\
\end{array}$ & $\begin{array}{l}\text { Selected features are mistakenly } \\
\text { considered as edge. }\end{array}$ \\
\hline $\begin{array}{l}\text { N. Senthilkumaran } \\
\text { et.al. [30] }\end{array}$ & $\begin{array}{l}\text { Ant Colony } \\
\text { Optimization }\end{array}$ & $\begin{array}{c}\text { Used as a great edge } \\
\text { enhancement method for the } \\
\text { already detected edges. }\end{array}$ & $\begin{array}{c}\text { Works relatively slow, Selected } \\
\text { features are mistakenly considered as } \\
\text { edge. }\end{array}$ \\
\hline $\begin{array}{l}\text { Jander Moreian } \\
\text { et.al. [39] }\end{array}$ & $\begin{array}{l}\text { Artificial Neural } \\
\text { Network }\end{array}$ & \begin{tabular}{|c|} 
Trained using specific patterns, \\
edges are selected more smoothly
\end{tabular} & $\begin{array}{c}\text { Many of the edges that are usually } \\
\text { found by other edge detection methods } \\
\text { are not considered as edges. }\end{array}$ \\
\hline $\begin{array}{c}\text { Raj Kumar Mohanta } \\
\text { et.al. [38] }\end{array}$ & Genetic Algorithm & $\begin{array}{l}\text { Selected features are correctly } \\
\text { considered as edges }\end{array}$ & $\begin{array}{c}\text { Works relatively slow, Selected } \\
\text { features are mistakenly considered as } \\
\text { edge, Complex Computation }\end{array}$ \\
\hline
\end{tabular}

\section{ANALYSIS OF THE REVIEW}

In previous sections, the classical and Soft Computing based Techniques for Edge Detection Problems are detailed. In this section, these techniques are analysed based on various performance parameters. The details are given in Table 1. It can be summed-up as under:

The classical techniques are simple but are inaccurate at times and sensitive to noise.

- $\quad$ Soft Computing Techniques are more accurate and promising than the classical techniques.

- The simple Soft Computing Techniques such as Genetic Algorithm, Fuzzy Logic, Swarm Intelligence, ACO, etc. also shows certain limitations.

- So, the current trend is to use mix two or more Soft Computing Techniques.

- Hybrid Soft Computing Techniques such as
Neuro-fuzzy, Fuzzy-GA, Memetic Algorithm [13], etc. have already shown promise.

- More Hybrid Soft Computing Techniques are yet to be tried for edge detection problem.

- Reducing the computational time of Soft Computing Techniques shall be another area for research.

\section{CONCLUSION}

The paper has tried to expend encroachment of Soft Computing Techniques to Edge Detection Problem in literature. All the techniques detailed in the paper try to provide optimal edge detection. The step edges model imposes two conditions that the transition is abrupt and the intensity is kept constant. The detectors described do identify the objects, circles, lines etc. Still the detectors miss the real edges and produce unsatisfactory results. Errors may be caused due to image characteristics, the wrong selection of algorithms and the implementation methods. In order to overcome the unexpected results and 
annoying noisy images each algorithm is used such that the signal to noise ratio for that image is higher. There are a number of Soft Computing Techniques for edge detection is available globally but not a particular method is present that can be universally accepted. It is a fact that a number of factors define and influence the process of edge detection; some of them are like texture, content of image, homogeneity of images, spatial characteristics and many more. New researches are in progress by hybridization of the two techniques for the betterment and obtaining an efficient technique for the edge detection.

\section{REFERENCES}

[1] Akanksha Bali, Shailendra Narayan Singh, "A Review on the Strategies \& Techniques of Image Segmentation", International Conf. on Intelligent Computing Applications, 2015, IEEE. DOI 10.1109/ICICA.2014.53,113-120.

[2] N. Anandakrihnan, S. Santhosh Baboo, "An Evaluation of Popular Edge Detection Techniques in Digital Image Processing”, International Conference on Intelligent Computing Applications (ICICA), 2014 IEEE DOI 10.1109/ICICA.2014.53,213-217.

[3] P. Thakur, N. Madaan, "A Survey of Image Segmentation Techniques," International Journal of Research in Computer Application and Robotics, ISSN: 2320-7345, Vol. 2, Issue 4, pp.158-165, April 2014.

[4] G.K. Seerha, R. Kaur, "A Study of Automatic Image Segmentation Techniques," ISSN: 2277 128X, 0018- , Vol. 3, Issue 2, pp. 435-437, Feb 2013.

[5] M. Weingart, O. Vascan, "Image Segmentation Processing-some Techniques and Experimental Results," 978-1-4799-2442-4/13 @2013 IEEE.

[6] Prakash B Metre, K. R. Radhika, Gowrishankar, "Survey of Soft Computing Techniques for Joint Radio Resource Management," 978-1-4799-0356-6/13 (C)2013 IEEE, pp. 1109-1112.

[7] H. P. Narkhede, "Review of Image Segmentation Techniques", International Journal of Science and Modern Technology, ISSN: 2319-6386, Vol.1, Issue 8, pp. 54-61, 2013.

[8] W. Khan, "Survey of Image Segmentation Techniques," Journal of Image and Graphics, Vol. 1, No. 4, pp. 166-170, Dec 2013.

[9] Jamil A. M. Saif, Ali Abdo Mohammed Al-Kubati, Abdultawab Saif Hazaa, and Mohammed Al-Moraish, "Image Segmentation Using Edge Detection and Thresholding," The 13th International Arab Conference on Information Technology, ACIT'2012 Dec.10-13 ISSN: 1812-0857.

[10] Kritika Sharma, Chandrashekhar Kamargaonkar, and Monisha Sharma, $\mathrm{PhD}$ "An Improved Image Segmentation Algorithm Based on Otsu Method," International Journal of Engineering Research \& Technology (IJERT), Vol. 1 Issue 6, August - 2012 ISSN: 2278-0181.

[11] A. Khanna, Dr. M. Shrivastava, "Unsupervised Techniques of Segmentation on Texture Images: A Comparison," 978-1-4673-1318-6/12 @2012 IEEE.
[12] Naveen Dagar, Rakhi Antil, Pawan Kumar Dahiya, "Improvised Canny Edge Detector \& Performance Metric of Edge Detector Using WPR (White Pixel Ratio)," IOSR Journal of Engineering, Vol. 3, No. 7, July 2013.

[13] Shivani Rathi, Pawan Kumar Dahiya, Pardeep Kumar, "Edge Detection using Memetic Algorithm," International Journal for Research in Applied Science and Engineering Technology, Vol. 1, No.3, pp. 42-52, October 2013.

[14] Pawan Kumar Dahiya, "Recent Trends in Evolutionary Computation," Ph.D. dissertation, ECE Dept., M. D. Univ., Rohtak, Haryana, India, 2011.

[15] N. Sharma, M. Mishra, M. Shrivastava, "Colour Image Segmentation," International Journal of Scientific and Technology, ISSN: 2277-8616, Vol.1, Issue 4, pp. 912, May 2012.

[16] Fahd Mohsen, Mohiy hadhoud, Kamel Mostafa, Khalid Amin, "A New Image Segmentation method based on new Particle Swarm Optimization," International Arab Journal of Information Technology, Vol. 9, No. 5, September 2012.

[17] Anand Gupta, Ravi Kumar Dalal, Rahul Gupta, Pulkit Wadhwa, "DGW-Canny: An Improvised Version of Canny Edge Detector," International Symposium on Intelligent signal Processing \& Communication System (ISPACS), Dec.7-9, 2011.

[18] Mr. Salem Saleh Al-Amri, Dr. N.V. Kalyankar and Dr. Khamitkar S.D, "Image Segmentation by using Edge Detection," International Journal on Computer Science and Engineering, Vol. 02, No. 03, 2010, 804807.

[19] Shriram K Vasudevan, "Automotive Image Processing Technique," International Journal of Engineering Science and Technology, Vol. 2 (7), 2010, 2632-2644, ISSN 0975-5462.

[20] K. Padma Vasavi, N. Udaya Kumar, E. V. Krishna Rao and M. Madhavi Latha "A Novel Statistical Thresholding in Edge Detection Using Laplacian Pyramid and Directional Filter Banks" Proceedings of the World Congress on Engineering and Computer Science, October 2010 Vol. I ISSN: 2078-0966.

[21] K. K. Singh, A. Singh, "A Study of Image Segmentation Algorithms for Different Types of Images," International Journal of Computer Science Issues, ISSN: 1694-0784, Vol.7, Issue 5, 2010.

[22] B.C. Wei, R. Mandava, "Multi objective Optimization Approaches in Image Segmentation- the Direction and Challenges", ICSRS Publication, Vol.2, pp. 41-65, 2010.

[23] R. Szeliski, Computer Vision: Algorithms and Applications, $1^{\text {st }}$ edition, Springer, pp. 265-303, 2010.

[24] S.S Varshney, N.Rajpal, R. Purwar, "Comparative study of Image Segmentation Techniques and Object Matching using Segmentation," International Conference on Methods and Models in Computer Science, 2009.

[25] N. Senthilkumaran and R. Rajesh, "Edge Detection Techniques for Image Segmentation - A Survey of Soft 
Computing Approaches", International Journal of Recent Trends in Engineering, Vol.1, No.2, May 2009, pp.250-254.

[26] N. Senthilkumaran and R. Rajesh, "A Study on Edge Detection Techniques for Image Segmentation", International Conference on Mathematics and Computer Science, (ICMCS-2009), January 2009, pp. 255-259.

[27] Amir Atapour Abarghouei, Afshin Ghanizadeh and Siti Mariyam Shamsuddin, "Advances of Soft Computing Methods in Edge Detection," IJASCA, vol 1, No 2, Nov. 2009.

[28] Susmita Ghosh, Swarnajyoti Patra, and Ashish Ghosh, "An unsupervised context-sensitive change detection technique based on modified self-organizing feature map neural network," International Journal of Approximate Reasoning 50 (2009) 37-50 January 2008.

[29] H. Zhang, J. E. Fritts, S. A. Goldman, "Image Segmentation Evaluation: A Survey of unsupervised methods," Computer Vision and Image Understanding, pp. 260-280, 2008.

[30] D. Kelkar, S. Gupta, "Improved Quadtree Method for Split Merge Image Segmentation", Emerging Trends in Engineering and Technology, 978-0-7695-32677/08 @2008 IEEE, pp. 44-47, 2008.

[31] Jing Tian, Weiyu Yu, and Shengli Xie, "An Ant Colony Optimization Algorithm for Image Edge Detection", IEEE, 78-1-4244-1823-7/08/\$25.00, 2008.

[32] N. Senthilkumaran and R. Rajesh, "Edge Detection Techniques for Image Segmentation - A Survey," Proceedings of Intl. Conf. on Managing Next Generation Software Applications, (MNGSA-08), Dec. 2008, pp.749-760.

[33] Mantas Paulinas and Andrius Usinskas, "A Survey of Genetic Algorithms Applicatons for Image Enhancement and Segmentation," Information Technology and Control, Vol.36, No.3, 2007, pp.278284.
[34] Mantas Paulinas and Andrius Usinskas, "A Survey of Genetic Algorithms Applicatons for Image Enhancement and Segmentation," Information Technology and Control, Vol.36, No.3, 2007, pp.278284

[35] Kanchan Deshmukh and G. N. Shinde, "An adaptive neuro-fuzzy system for color image segmentation," $J$. Indian Inst. Sci., vol. 86, Sept.-Oct.2006, pp.493-506.

[36] M. Gandomkar, M. Vakilian and M. Ehsan, "A Combination of Genetic Algorithm and Simulated Annealing for Optimal Distributed DG Allocation in Distributed Networks," Proceedings of the IEEE Electrical and Computer Engineering Canadian Conference, 2005.

[37] M. Abdulghafour, "Image segmentation using Fuzzy logic and genetic algorithms," Journal of WSCG, vol.11, no. 1, 2003.

[38] H.D Chung, Y. Sun, “A Hierarchial Approach to Color Image Segmentation, "1057-7149/00 @ 2000 IEEE, Vol. 9, No. 12, pp. 2071-2083.

[39] J. Shi and J. Malik, "Normalized Cuts and Image Segmentation", IEEE.

[40] Raj Kumar Mohanta, Binapani Sethi, "A Review of Genetic Algorithm application for Image Segmentation," Raj Kumar Mohanta et al, Int. J. Computer Technology \& Applications, Vol 3 (2), 720723,2000 .

[41] Jander Moreira and Luciano Da Fontoura Costa, "Neural-based color image segmentation and classification using self-organizing maps",Anais do IX SIBGRAPI, 1996, pp.47-54.

[42] Andreas Koschan, "A Comparative Study On Color Edge Detection", Reprint from Proceedings 2nd Asian Conference on Computer Vision ACCV'95, Singapore, 5-8 December 1995, Vol. III, pp. 574-578.

[43] Suchendra M. Bhandarkar, Yiqing Zhang and Walter D. Potter "An Edge Detection technique using genetic algorithm-based optimization" Vol. 27, no. 9, pp. 1159-1180, March 1994. 